\title{
ENFERMAGEM NA PREVENÇÃO DE INFECÇÃO EM CATETER TOTALMENTE IMPLANTADO NO PACIENTE ONCOLÓGICO
}

\author{
Thais Fernandes de Oliveira ${ }^{1}$, Maria Cristina Soares Rodrigues ${ }^{2}$
}

\begin{abstract}
RESUMO: O objetivo dessa comunicação livre é apontar evidências científicas acerca de contribuições da Enfermagem na prevenção de infecção em cateter totalmente implantado de pacientes oncológicos, com base em literatura de referência acerca do assunto. Os estudos foram selecionados entre novembro de 2015 e janeiro de 2016, nas bases de dados Cochrane Library, Literatura Latino-Americana e do Caribe em Ciências da Saúde, Medical Literature Analysis and Retrieval System Online via PubMed e Web of Science, por meio dos descritores cateter de permanência, cuidados de enfermagem, segurança do paciente. As evidências foram organizadas em três categorias: punção segura do cateter totalmente implantado, segurança na troca e manutenção do curativo do cateter totalmente implantado, e administração segura de medicamentos e manutenção do sistema fechado. A assistência de enfermagem ao paciente portador de cateter totalmente implantado é cercada de riscos que podem resultar na infecção do cateter, porém, a existência de ações preventivas é capaz de reduzir esses riscos. DESCRITORES: Infecções relacionadas a cateter; Cuidados de enfermagem; Segurança do paciente.
\end{abstract}

\section{NURSING IN THE PREVENTION OF INFECTION IN TOTALLY IMPLANTED CATHETERS OF ONCOLOGICAL PATIENTS}

\begin{abstract}
The aim of this free communication is to highlight scientific evidence related to nursing contributions in preventing infection in totally implanted catheters of oncological patients, based on reference literature on the subject. The studies were selected between November 2015 and January 2016, in the Cochrane Library, Latin American and Caribbean Center on Health Sciences Information, and Medical Literature Analysis and Retrieval System Online databases via PubMed and Web of Science, using the descriptors indwelling catheter, nursing care, and patient safety. The evidence was organized into three categories: safe puncture of the totally implanted catheter, safety in the change and maintenance of the totally implanted catheter dressing, and safe administration of medication and maintenance of the closed system. Nursing care for totally implanted catheter patients is surrounded by risks that can result in infection of the catheter, however, the existence of preventive measures can reduce these risks. DESCRIPTORS: Catheter related infections; Nursing care; Patient safety.
\end{abstract}

\section{ENFERMERÍA EN LA PREVENCIÓN DE INFECCIÓN EN CATÉTER TOTALMENTE IMPLANTADO EN PACIENTE ONCOLÓGICO}

RESUMEN: Fue objetivo de esta comunicación libre apuntar evidencias científicas acerca de contribuciones de la Enfermería en la prevención de infección en catéter totalmente implantado de pacientes oncológicos, basándose en literatura de referencia respecto al asunto. Los estudios fueron seleccionados entre noviembre de 2015 y enero de 2016, en las bases de datos Cochrane Library, Literatura Latinoamericana y del Caribe en Ciencias de la Salud, Medical Literature Analysis and Retrieval System Online por PubMed y Web of Science, utilizándose los descriptores catéter de permanencia, cuidados de enfermería, seguridad del paciente. Las evidencias fueron organizadas en tres categorías: punción segura del catéter totalmente implantado, seguridad en el cambio y en la manutención de las vendajes del catéter totalmente implantado, y administración segura de medicamentos y manutención del sistema cerrado. La asistencia de enfermería al paciente portador de catéter totalmente implantado presenta riesgos que pueden resultar en infección del catéter, pero la existencia de acciones preventivas es capaz de reducir esos riesgos.

DESCRIPTORES: Infecciones asociadas a catéter; Cuidados de enfermería; Seguridad del paciente.

${ }^{1}$ Enfermeira. Mestranda em Enfermagem Universidade de Brasília. Brasília, DF, Brasil.

${ }^{2}$ Enfermeira. Pós-doutora em Ciências da Saúde. Docente de Enfermagem da Universidade de Brasília. Brasília, DF, Brasil.

Autor Correspondente:

Thais Fernandes de Oliveira

Universidade de Brasília - Faculdade de Ciências da Saúde, Campus Universitário Darcy Ribeiro

70910-900 Asa Norte, Brasília, DF, Brasil

E-mail: thaisoliveiraenf0911@gmail.com
Recebido: 28/02/2016

Finalizado: 16/06/2016 


\section{- INTRODUÇÃO}

A segurança do paciente advém da necessidade de fornecer subsídios para uma assistência segura e de qualidade nos serviços de saúde. Entende-se por segurança do paciente (SP) ações que visam reduzir a um mínimo aceitável, o risco de dano desnecessário associado ao cuidado de saúde ${ }^{(1)}$.

Nos últimos anos, a Organização Mundial da Saúde (OMS) incentivou a adoção de práticas seguras na assistência à saúde. Uma estratégia foi a implentação das Metas Internacionais de Segurança do Paciente, destacando-se aquela que visa prevenir as infecções relacionadas à assistência à saúde (IRAS). As IRAS são responsáveis por danos aos pacientes, internação prolongada e altos custos aos sistemas de saúde ${ }^{(2)}$. Nessa situação, o dano ao paciente na maioria dos casos é irreversível e inevitável.

Os riscos envolvidos na prestação de cuidado em saúde possuem particularidades a depender do perfil clínico do paciente; dentre eles, tem-se o paciente oncológico, que é cercado de riscos ao longo de todo o tratamento. Esses riscos envolvem a própria doença de base (câncer), o tratamento e a assistência.

Câncer, definido como a multiplicação desordenada de determinado tipo celular, tem o potencial de causar danos locais ou sistêmicos. As opções terapêuticas são quimioterapia, cirurgia, radioterapia. A quimioterapia é uma das opções mais promissoras, pois a sua ação sistêmica é capaz de induzir a morte das células cancerígenas em todos os órgãos e tecidos atingidos pela doença ${ }^{(3-4)}$.

Os riscos relacionados ao cuidado em saúde são os mais preocupantes, uma vez que podem ser evitados. Quando a terapêutica empregada é a quimioterapia, os pacientes necessitam de uma via segura para a administração das drogas, dado que a administração em vias periféricas leva à falência da rede venosa ${ }^{(2)}$.

O cateter totalmente implantado (CVC-TI) é uma opção de cateter de longa permanência que permite a infusão de agentes quimioterápicos, soluções, transfusões sanguíneas, nutrição e coleta de exames de forma segura ${ }^{(5)}$. A segurança do paciente oncológico é permissa básica para uma assistência de qualidade, visto que a doença, o tratamento e as complicações advindas do mesmo tornam o paciente frágil e exposto a riscos constantes.

Os cuidados de enfermagem diante de um paciente oncológico portador de CVC-TI visam à adoção de medidas que impossibilitem a ocorrência de infecção no cateter. Reduzir o risco de infecções associadas aos cuidados de saúde é meta internacional de segurança do paciente ${ }^{(6)}$. Essas metas foram determinadas pela OMS a fim de reduzir os erros relacionados à assistência em saúde e dessa forma garantir ao paciente uma permanência segura no ambiente hospitalar.

Ressalta-se que o uso de dispositivos intravenosos, como o CVC-TI, tem o potencial de causar dano ao paciente e está associado à morbidade e mortalidade, especialmente durante a internação hospitalar ${ }^{(7)}$. Dentre esses danos, destaca-se a infecção do CVC-TI, que é uma complicação responsável pela descontinuidade do tratamento, a resistência antimicrobiana dos pacientes imunossuprimidos com grande risco para o desenvolvimento de $\operatorname{sepse}^{(7)}$.

A infecção do CVC-TI é uma complicação considerada tardia, pois ocorre após com o uso, ou seja, a partir da colonização da pele, utilização de material contaminado e por contaminação das conexões do cateter ${ }^{(8)}$.

Considerando a relevância do tema, este texto tem por objetivo apontar evidências científicas acerca das contribuições de enfermagem na prevenção de infecção em CVC-TI de pacientes oncológicos. Os dados foram obtidos por meio da busca na base de dados: Cochrane Library, Literatura Latino-Americana e do Caribe em Ciências da Saúde (LILACS), Medical Literature Analysis and Retrieval System Online (MEDLINE) via PubMed e Web of Science, sendo incluídos somente aqueles publicados entre 2011 e 2015, e para tanto foram utilizados os descritores cateter de permanência, cuidados de enfermagem, segurança do paciente. A coleta de dados ocorreu no período de novembro de 2015 a janeiro de 2016.

As evidências foram sintetizadas em três categorias, a seguir apresentadas: punção do cateter totalmente implantado, administração de medicamentos e manutenção do sistema fechado, e troca do curativo. 


\section{- PUNÇÃO SEGURA DO CATETER TOTALMENTE IMPLANTADO}

A punção do CVC-TI é atribuição do enfermeiro. Exige conhecimento teórico e habilidade na técnica estéril. O acesso ao CVC-TI dá-se por meio de agulha tipo Hubber cujo bisel lateralizado aumenta a meia vida do cateter ${ }^{(2)}$. A antissepsia da pele com clorexidina alcoólica é a maneira mais efetiva de preparo da pele para a punção, pois sua ação microbicida mantém efeito residual na pele por tempo superior ao álcool e polvidine alcoólico e dessa forma reduz o risco de infecção relacionado à punção do CVC-Tl ${ }^{(3,9-10)}$.

Além da antissepsia da pele a ser puncionada, o correto preparo material a ser utilizado (máscara cirúrgica simples, gorro, gaze e luva estéril, agulha tipo Hubber) são medidas importantes na prevenção de infecção do CVC-TI ${ }^{(10)}$.

A técnica estéril é a primeira barreira de segurança para o paciente portador de CVC-TI, pois impede que microrganismos invadam o óstio ou o túnel do cateter resultando em infecção e sepse. É necessário que o enfermeiro, ao puncionar o CVC-TI, tenha consciência que a sua função durante o procedimento não é apenas garantir a infusão de medicamentos por aquela via, mas garantir que a técnica aplicada é segura.

\section{SEGURANÇA NA TROCA E MANUTENÇÃO DO CURATIVO DE CVC-TI}

A troca do curativo do CVC-TI exige escolha correta da cobertura, avaliação do óstio do cateter, identificação precoce de sinais flogistícos e o cumprimento na periodicidade da troca do curativo.

O curativo serve com proteção para o local de inserção da agulha. A clorexidina alcoólica é a solução mais indicada para antissepsia da região devido ao seu efeito residual. Quanto ao intervalo entre a troca do curativo, considera-se que quando a cobertura utilizada for gaze estéril, a troca deverá ser realizada a cada 48 horas, e quando a película transparente for a escolha, a troca deverá ser realizada a cada $7 \operatorname{dias}^{(9)}$.

Cabe destacar que o momento da troca do curativo é circunstância ímpar para avaliação quanto à presença de sinais flogistícos. Além disso, a revisão diária do cateter evita atrasos desnecessários na retirada de linhas que não estão sendo utilizadas, pois a o risco de infecção é proporcional ao tempo que o cateter permanece. Recomenda-se a cobertura de filme transparente, pois permite a visualização precoce de sinais flogistícos ${ }^{(10)}$.

\section{- ADMINISTRAÇÃO SEGURA DE MEDICAMENTOS E MANUTENÇÃO DO SISTEMA FECHADO}

As conexões ligadas ao CVC-TI devem ser rigorosamente manuseadas para evitar contaminação direta ou indireta do paciente por microrganismos ${ }^{(7)}$. A administração de medicamentos, dentre os quais os quimioterápicos, são administrados com mais frequência, requer higienização das mãos imediatamente antes do contato e antissepsia com álcool a $70 \%$ das conexões ${ }^{(3)}$. Alguns microrganismos responsáveis por infecções provenientes da utilização de um CVC-TI são oriundas das mãos dos profissionais que manipulam este dispositivo ${ }^{(11)}$.

Manter as conexões do cateter desprotegidas favorece a colonização do óstio e do lúmen por bactérias da flora do paciente ou das mãos do profissional, além de bactérias do meio externo, como as multirresistentes presentes nos hospitais ${ }^{(11)}$. A troca do equipo deve realizada em um intervalo não superior a 96 horas $^{(10)}$.

As ações de enfermagem referentes a essa etapa do processo de trabalho são simples e não requerem equipe completa, materiais e insumos, apenas o reconhecimento da equipe de que a lavagem e a antissepsia das mãos, a desinfecção dos injetores laterais e conexões são ações capazes de salvar vidas. 


\section{- IMPLICAÇÕES DAS AÇÕES DE ENFERMAGEM NA SEGURANÇA DO PACIENTE PORTADOR DE CVC-TI}

As ações de enfermagem descritas neste texto informativo implicam diretamente em cuidados que visam reduzir a um mínimo aceitável o risco dos pacientes portadores de CVC-TI ter infecção decorrentes do cuidado em saúde. Essas ações, apesar de simples, exigem da equipe de enfermagem a adoção de medidas com intuito de evitar que os microrganismos relacionados ao manuseio do cateter ou da própria microbiota do paciente tornem o cateter inutilizado devido à infecção, bem como interromper ou adiar o tratamento ou levar à sepse e ao óbito.

\section{CONSIDERAÇÕES FINAIS}

As evidências das contribuições da enfermagem apontadas neste artigo mostram que a punção segura, a manutenção e troca do curativo e a manutenção do sistema fechado são cuidados imprescindíveis para a prevenção de infecção do cateter totalmente implantado. Essas ações visam a uma assistência segura para o paciente oncológico, já tão fragilizado pela doença e tratamento.

Espera-se que esse texto sinalize aos profissionais da enfermagem que as ações apontadas são simples e capazes de evitar danos ao paciente. Além disso, almeja-se que novas pesquisas na mesma temática possam ser desenvolvidas com abordagens metodológicas diferentes.

\section{REFERÊNCIAS}

1. Ministério da Saúde (BR). Documento de referência para o Programa Nacional de Segurança do Paciente. Brasília (DF): Ministério da Saúde; 2014.

2. Gomes AR, Sá SPC. Perfil dos pacientes e cateteres venoso central totalmente implantado de um hospital de oncologia. Rev enferm UFPE online. [Internet] 2014; 8(7) [acesso em 05 dez 2015]. Disponível: http://www.revista. ufpe.br/revistaenfermagem/index.php/revista/article/download/6143/9453.

3. Bonassa EMA, Gato MIR. Terapêutica oncológica para enfermeiros e farmacêuticos. $4^{a}$ ed. São Paulo: Atheneu; 2012.

4. Brasil. Instituto Nacional do Câncer (INCA). [Internet] Câncer: o que é. Rio de Janeiro: Instituto Nacional do Câncer [acesso em 10 dez 2015]. Disponível: http://www2.inca.gov.br/wps/wcm/connect/cancer/site/oquee.

5. Bruzi LM, Mendes DC. The importance of nursing care in managing complications related to fully-implantable catheters. Rev. esc. enferm. USP. [Internet] 2011; 45(2) [acesso em 10 dez 2015]. Disponível: http://dx.doi. org/10.1590/S0080-62342011000200031.

6. Martins M. Qualidade do cuidado em saúde. In: Sousa P, organizadores. Segurança do paciente: conhecendo os riscos nas organizações de saúde. Rio de Janeiro: EaD/ENSP; 2014. p.140.

7. Menezes VPS, Bittencourt AR, Menezes MFB. Infection related to central venous catheter: indicator of quality in oncology. J. Res.: fundam. care. online. [Internet] 2013; 5(3) [acesso em 05 dez 2015]. Disponível: https://dx.doi. org/10.9789/2175-5361.2013v5n3p373.

8. de Oliveira EB, Reis MA, Avelar TM, Vieira SC. Cateteres venosos centrais totalmente implantáveis para quimioterapia: experiência com 793 pacientes. Rev. Col. Bras. Cir. [Internet] 2013; 40(3) [acesso em 15 dez 2015]. Disponível: http://dx.doi.org/10.1590/S0100-69912013000300004.

9. Pires NN, Vasques Cl. Knowledge regarding the handling of the totally-implanted venous access device. Texto Contexto Enferm. [Internet] 2014; 23(2) [acesso em 05 dez 2015]. Disponível: http://dx.doi.org/10.1590/010407072014000830013.

10. Henrique DM, Tadeu CN, Alves FH, Trindade LPC, Fernandes MSR, Macedo ML, et al. Fatores de risco e recomendações atuais para prevenção de infecção associada a cateteres venosos centrais: uma revisão de 
literatura. Rev Epidemiol Control Infect. [Internet] 2013; 3(4) [acesso em 10 dez 2015]. Disponível: http://dx.doi. org/10.17058/reci.v3i4.4040.

11. dos Santos SF, Viana RS, Alcoforado CLG, Campos CC, de Matos SS, Ercole FF. Ações de enfermagem na prevenção de infecções relacionadas ao cateter venoso central: uma revisão integrativa. Rev. SOBECC. [Internet] 2014; 19(4) [acesso em 12 dez 2015]. Disponível: http://itarget.com.br/newclients/sobecc.org.br/2015/pdfs/v19n4/ SOBECC_v19n4_219-225.pdf. 